\title{
One-Year Outcome of Aflibercept and Photodynamic Therapy in a Caucasian Patient with Polypoidal Choroidal Vasculopathy Refractory to Ranibizumab and Photodynamic Therapy
}

\author{
Marta Medina-Baena ${ }^{a} \quad$ María Jesús Huertos-Carrillo ${ }^{a} \quad$ Laura Rodríguez $^{b}$ \\ Juan Ignacio García-Pulido ${ }^{a}$ Carlos Cornejo-Castillo ${ }^{b}$ \\ José María Calandria-Amiguetti ${ }^{\text {b }}$ \\ a Unidad de Retina y Vítreo, Hospital Universitario Puerto Real, Cádiz, Spain; ${ }^{\text {b Unidad de }}$ \\ Retina y Vítreo, Hospital Universitario Puerta del Mar, Cádiz, Spain
}

\section{Keywords}

Aflibercept · Polypoidal choroidal vasculopathy · Polypoidal lesion · Combination therapy . Photodynamic therapy

\begin{abstract}
Polypoidal choroidal vasculopathy (PCV) is a subtype of neovascular age-related macular degeneration characterised by an abnormal branching vascular network with aneurysmal polypoidal choroidal vascular lesions. PCV is more prevalent in Asian populations than in Caucasians, which may explain its underdiagnosis in Western countries. Evidence regarding the efficacy of different anti-vascular endothelial growth factor (anti-VEGF) agents on PCV is scarce, with most of these studies being conducted in Asian treatment-naïve patients. Ranibizumab was the first anti-VEGF agent to demonstrate the superiority of a combination of photodynamic therapy (PDT) and anti-VEGF over PDT or anti-VEGF monotherapy for inducing polyp regression in Asian patients with PCV. The efficacy of other anti-VEGF agents has been less studied. Resistance to ranibizumab has been described. Aflibercept offers another mech-
\end{abstract}




\section{Case Reports in Ophthalmology}

Case Rep Ophthalmol 2018;9:172-178 DOI: $10.1159 / 000487227$

C 2018 The Author(s). Published by S. Karger AG, Basel www.karger.com/cop

Medina-Baena et al.: One-Year Outcome of Aflibercept and PDT in a Caucasian Patient with PCV Refractory to Ranibizumab and Photodynamic Therapy

anism of targeting choroidal neovascular lesions. A 75-year-old Caucasian woman presenting to our office was diagnosed with PCV using indocyanine green angiography. Combination therapy with a loading dose of $0.5 \mathrm{mg}$ intravitreal ranibizumab followed by PDT at standard fluence at month 4 and a fourth dose of ranibizumab at month 5 yielded no visual or anatomic outcomes. Treatment was switched to intravitreal aflibercept at month 6 (3 monthly loading doses of $2.0 \mathrm{mg}$ ) followed by half-fluence PDT (month 9). Optical coherence tomography revealed remission of the anatomic lesions. Right-eye visual acuity increased to 0.6. Aflibercept injections were administered bimonthly afterwards. Follow-up during 1 year has shown functional and anatomic stability.

(C) 2018 The Author(s)

Published by S. Karger AG, Basel

\section{Introduction}

Polypoidal choroidal vasculopathy (PCV) is a subtype of neovascular age-related macular degeneration (AMD) characterised by an abnormal branching vascular network with aneurysmal polypoidal choroidal vascular lesions $[1,2]$. This can lead to serous or haemorrhagic pigment epithelial detachment (PED), episodes of exudative retinal detachment and subretinal exudation and haemorrhage [3]. Repeated bleeding and leakage lead to retinal pigment epithelium and photoreceptor degeneration, scarring and reversible visual loss in about half of the patients [2].

PCV occurs in middle-aged to elderly people of both sexes, although its prevalence varies among different ethnic groups, with pigmented ones - Asians and Blacks - showing a higher prevalence than Caucasians [2]. Thus, while PCV is the most common subtype of AMD in Asia, choroidal neovascularization secondary to AMD (CNV-AMD) is the typical subtype in Western populations [2]. Both subtypes share many clinical features and risk factors but differ in natural history and, most importantly, treatment approaches and outcomes - especially with regard to anti-vascular endothelial growth factors (anti-VEGFs) [2]. All this has increasingly raised awareness of PCV among Caucasians, and accurate diagnosis is of utmost importance. The increasing use of indocyanine green angiography (ICGA), the gold standard for PCV diagnosis [1], in routine practice in Western healthcare settings is helping to reduce the underdiagnosis of PCV.

PCV has been shown to vary clinically among ethnic populations [4]. PCV has mostly been described in Asiatic populations, and most of the studies evaluating the efficacy of different anti-VEGF agents, besides being limited in number [1, 2], have been conducted in Asiatic populations and predominantly in treatment-naïve patients. More evidence is needed about the efficacy of anti-VEGF agents in Caucasian populations, including among those refractory to previous anti-VEGF treatments. We report a case of an elderly Caucasian (Spanish) woman presenting with PCV refractory to combined therapy with intravitreal ranibizumab and photodynamic therapy (PDT) who was successfully treated with intravitreal aflibercept and PDT.

\section{Case Report}

A 75-year-old woman presented to our office in February 2015 for a routine check-up. On examination, visual acuity was 0.6 and 0.8 in the right and left eyes, respectively. Fundus examination revealed the presence of scattered drusen indicative of AMD in both eyes. Optical coherence tomography (OCT) dismissed the presence of neovascular membrane. The 
patient was provided with an Amsler grid and instructed in its use. A follow-up visit was scheduled for 6 months later.

The patient returned 5 months later (July 2015) because she had noticed irregularities when testing her eyes. Visual acuity had remained stable. Fundus examination confirmed the persistence of drusen and revealed a juxtapapillary haemorrhage in the superior temporal arcade of the right eye. A new OCT showed an area of subretinal fluid and a PED (Fig. 1a). Given the suspicion of PCV, fluorescein angiography (FA) was carried out, which showed the presence of significant PEDs in the perifoveal capillary network (venous phase). No polyps were observed. An ICGA was scheduled to confirm PCV. Meanwhile, intravitreal 0.5-mg ranibizumab therapy at 4-week intervals was started.

The patient was assessed after the 3 monthly loading doses of ranibizumab (October 2015). Visual acuity of the right eye had decreased to 0.4. OCT revealed persistent PED under a sensorineural detachment area in the superior temporal arcade. An ICGA, performed shortly before, confirmed the existence of 3 polypoidal lesions in the superior papillary area of the right eye (Fig. 1b). After confirmation of the diagnosis of PCV, PDT with verteporfin was administered at standard fluence $\left(50 \mathrm{~J} / \mathrm{cm}^{2}, 300 \mathrm{~mW} / \mathrm{cm}^{2}, 83 \mathrm{~s}\right.$, dose $6 \mathrm{mg} / \mathrm{m}^{2}$; diameter: $6,400 \mu \mathrm{m}$ ). A 4 th dose of ranibizumab was administered 4 weeks later (November 2015).

At a follow-up visit 4 weeks later (December 2015), OCT scans showed no improvement in visual acuity or anatomic benefits (Fig. 2). Intravitreal anti-VEGF therapy with ranibizumab was switched to aflibercept $2.0 \mathrm{mg}$. Half-fluence PDT $\left(25 \mathrm{~J} / \mathrm{cm}^{2}, 300 \mathrm{~mW} / \mathrm{cm}^{2}, 83 \mathrm{~s}\right.$, dose $6 \mathrm{mg} / \mathrm{m}^{2}$; diameter: 5,000 $\mu \mathrm{m}$ ) was administered after the 3-monthly loading dose (March 2016). OCT examination 4 weeks later (April 2016) revealed the remission of the anatomic lesions (Fig. 3). Right-eye visual acuity increased to 0.6. Aflibercept injections have been administered bimonthly since then. Follow-up during 1 year (up to May 2017) has shown the functional and anatomic stability of our patient.

\section{Discussion}

A PCV diagnosis among Caucasians is still challenging, which has a profound effect on successful treatment and outcomes. In our patient, signs of AMD in the right eye and irregular elevations of the retinal pigment epithelium in the perifoveal capillary network seen on FA, which were compatible with areas of subretinal fluid leakage observed on OCT, led to a diagnosis of neovascular AMD. Given the inadequateness of FA to distinguish PCV from CNVAMD [3], ICGA imaging was needed for the differential diagnosis. This technique allowed the observation of 3 polypoidal lesions in the superior papillary area, which confirmed the diagnosis of PCV. The poor response to the anti-VEGF ranibizumab before the ICGA results were available was also suggestive of PCV, given that this agent is less effective in PCV compared to CNV-AMD [2].

PDT has shown to be effective in improving visual acuity and in inducing the regression of polyps among PCV patients $[2,3]$. However, this therapy is associated with a high rate of recurrence and the possibility of complications, such as haemorrhages and choroidal ischaemia $[2,3]$. PDT has a role in the treatment of extrafoveal polyps, but it is not recommended for the initial treatment of active juxtafoveal or subfoveal PCV [1]. The combination of PDT with an anti-VEGF agent has the benefit of targeting both components of the PCV complex (polypoidal lesions and the branching vascular network) [2]. Several studies have proven the superior efficacy of combined PDT + anti-VEGF agent over anti-VEGF agent alone 


\section{Case Reports in Ophthalmology}

with regard to polyp regression/closure and visual outcomes [2, 3]. The superiority of PDT in combination with an anti-VEGF agent or alone over the anti-VEGF agent alone was first confirmed in the prospective, randomized EVEREST study, conducted in Asian patients, where PDT combined with ranibizumab $0.5 \mathrm{mg}$ or alone showed greater efficacy in complete polyp regression at month 6 compared to ranibizumab $0.5 \mathrm{mg}$ monotherapy (77.8 vs. 71.4 vs. $28.6 \%$, respectively, $p<0.01$ ). The 3 treatments led to similar improvements in mean best-corrected visual acuity [5]. Moreover, it has been reported that PCV eyes resistant to ranibizumab monotherapy have been successfully treated (mostly achieving resolution of exudation) with PDT, anti-VEGF/PDT combination therapy or combined anti-VEGF monotherapy [6]. Treatment with the loading dose ranibizumab failed in our patient, on the basis of the loss of vision and persistence of PED as observed on OCT imaging. Addition of PDT followed by a fourth intravitreal injection did not translate into functional or anatomic (OCT) improvements at 1-month follow-up.

Alternatives to this therapy included switching to bevacizumab or aflibercept as part of the combined therapy with PDT. Evidence with bevacizumab is small, with most studies being limited by a short follow-up [2]. Moreover, resistance to bevacizumab has also been described [6]. Aflibercept offers another way of targeting choroidal neovascular lesions as it shows a higher binding affinity to VEGF A and B and a longer intravitreal half-life compared to other anti-VEGF agents. It also has the capacity to antagonize growth factors other than VEGF, such as placental growth factor, which is believed to play a role in PCV [7, 8]. The efficacy and safety of aflibercept monotherapy in PCV has been assessed in several case studies showing visual improvements and a regression of up to 69\% of polypoid lesions at 12 months, suggesting that aflibercept may be more effective at achieving polypoidal lesion closure than other anti-VEGF therapies [2, 9]. Evidence regarding the efficacy of combination therapy with aflibercept and PDT is scarce. A case series of 20 patients with PCV has shown that this combination provides visual and anatomic improvements over a 12-month followup period. A retrospective chart review comparing 1-year outcomes among 12 patients who received a loading dose of aflibercept (with [ $n=11]$ or without [ $n=12]$ PDT) followed by as needed injections has shown that although both treatments yielded similar visual and anatomic outcomes, patients receiving combination therapy required fewer injections [10]. Recently, the benefit of combined therapy with aflibercept $2.0 \mathrm{mg}$ and PDT compared to aflibercept $2.0 \mathrm{mg}$ monotherapy has been assessed in a randomised, double-masked, shamcontrolled, phase $3 \mathrm{~b} / 4$ study (PLANET study) conducted in 318 patients from 62 centres, mostly in Asia. In this study with a follow-up period of 52 weeks, aflibercept monotherapy showed substantial visual acuity gains (primary endpoint was change in best-corrected visual acuity ETDRS letters score from baseline to week 52) and anatomical benefits, with $>80 \%$ of patients showing no active polyps at week 52 , a $58-67 \%$ reduction in the mean area of polyps and with more than a third of patients experiencing complete polyp regression. Adding PDT did not appear to provide any additional functional benefit [11]. Despite these encouraging results, and as previously mentioned, it should be noted that all the above studies with aflibercept have been conducted in Asian treatment-naïve patients, meaning that - as with other anti-VEGF agents - there is a lack of evidence for the use of aflibercept in Western populations.

There is also paucity of evidence regarding the effectiveness of aflibercept in patients refractory to other anti-VEGF agents, which has also been mostly investigated in Asian populations. The EPIC study, a prospective, open-label study in patients with PCV and active haemorrhage, exudation or recent decrease in vision, included 11 patients (52\%) previously treated with ranibizumab or bevacizumab, in whom aflibercept monotherapy achieved 


\section{Case Reports in Ophthalmology}

Medina-Baena et al: One-Year Outcome of Aflibercept and PDT in a Caucasian Patient with PCV Refractory to Ranibizumab and Photodynamic Therapy

marked improvement in retinal PED and persistent polyps at 6-month follow-up [12]. Kawashima et al. [13] reported a significant reduction of central retinal thickness and an improvement in the logarithm of minimum angle of resolution 6 months after starting treatment with aflibercept in 26 Asian PCV patients refractory to ranibizumab. A case study in a single female patient (unknown ethnicity) has also demonstrated that intravitreal aflibercept in monotherapy may be efficacious in patients with PCV who are refractory to other treatments, including bevacizumab, ranibizumab, PDT, and PDT combination therapy [14].

To the best of our knowledge, the case reported here is the first one describing the efficacy of combined therapy of intravitreal aflibercept $2 \mathrm{mg}$ and PDT in a Caucasian patient refractory to combined therapy with ranibizumab and PDT. Given the lack of proper studies with aflibercept conducted in Caucasian PCV patients, it is currently unknown whether adding PDT to treatment for such patients would allow their receiving fewer injections or if it would yield greater visual and anatomic improvements. Should the latter be the case, PDT could be left to non-responders or to cases requiring too frequent injections, thus avoiding the complications associated with the use of PDT $[2,3]$. Further research is warranted to elucidate the role of aflibercept with and without PDT in Caucasian patients refractory to other anti-VEGF agents.

\section{Acknowledgements}

We thank Beatriz Viejo, PhD, for her assistance in the writing of the manuscript and editorial support. This service was supported by Bayer HealthCare, who had no influence on the interpretation of results. The views expressed are therefore based on the authors' opinions.

\section{Statement of Ethics}

The authors have no ethical conflicts to disclose.

\section{Disclosure Statement}

The authors declare no conflicts of interest.

\section{References}

1 Koh AH; Expert PCV Panel, Chen LJ, Chen SJ, Chen Y, Giridhar A, et al: Polypoidal choroidal vasculopathy: evidence-based guidelines for clinical diagnosis and treatment. Retina 2013;33:686-716.

$\checkmark 2$ Wong CW, Yanagi Y, Lee WK, Ogura Y, Yeo I, Wong TY, et al: Age-related macular degeneration and polypoidal choroidal vasculopathy in Asians. Prog Retin Eye Res 2016;53:107-139.

-3 Wong RL, Lai TY: Polypoidal choroidal vasculopathy: an update on therapeutic approaches. J Ophthalmic Vis Res 2013;8:359-371.

4 Shanta JG, Kokame GT: Polypoidal choroidal vasculopathy. Retina Today. 2016. http://retinatoday.com/2016/06/polypoidal-choroidal-vasculopathy/ (accessed September 28, 2017).

5 Koh A, Lee WK, Chen LJ, Chen SJ, Hashad Y, Kim H, et al: EVEREST study: efficacy and safety of verteporfin photodynamic therapy in combination with ranibizumab or alone versus ranibizumab monotherapy in patients with symptomatic macular polypoidal choroidal vasculopathy. Retina 2012;32:1453-1464.

6 Cho M, Barbazetto IA, Freund KB: Refractory neovascular age-related macular degeneration secondary to polypoidal choroidal vasculopathy. Am J Ophthalmol 2009;148:70-78.e1. 


\section{Case Reports in Ophthalmology}

\begin{tabular}{l|l}
\hline DOI: $10.1159 / 000487227$ & C 2018 The Author(s). Published by S. Karger AG, Basel \\
www.karger.com/cop
\end{tabular}
www.karger.com/cop

Medina-Baena et al:: One-Year Outcome of Aflibercept and PDT in a Caucasian Patient with PCV Refractory to Ranibizumab and Photodynamic Therapy
$>7$

Hosokawa M, Morizane Y, Hirano M, Kimura S, Kumase F, Shiode Y, et al: One-year outcomes of a treatand-extend regimen of intravitreal aflibercept for polypoidal choroidal vasculopathy. Jpn J Ophthalmol 2017;61:150-158.

-8 Inoue M, Arakawa A, Yamane S, Kadonosono K: Short-term efficacy of intravitreal aflibercept in treatment-naive patients with polypoidal choroidal vasculopathy. Retina 2014;34:2178-2184.

-9 Lee JE, Shin JP, Kim HW, Chang W, Kim YC, Lee SJ, et al: Efficacy of fixed-dosing aflibercept for treating polypoidal choroidal vasculopathy: 1-year results of the VAULT study. Graefes Arch Clin Exp Ophthalmol 2017;255:493-502.

10 Takayama K, Kaneko H, Kataoka K, Hattori K, Ra E, Tsunekawa T, et al: Comparison between 1-year outcomes of aflibercept with and without photodynamic therapy for polypoidal choroidal vasculopathy: retrospective observation study. PLoS One 2017;12:e0176100.

11 Mitchell P, Wong TY, Chen SJ, Zhang E, Ishibashi T, Leal S: Intravitreal aflibercept in the treatment of polypoidal choroidal vasculopathy: the planet study. Presented at the 17th EURETINA Congress, 7-10 September, Barcelona (Spain). 2017. http://www.euretina.org/barcelona2017/programme/freepapers-details.asp?id=13363\&day=0 (accessed September 28, 2017).

12 Kokame GT, Lai JC, Wee R, Yanagihara R, Shantha JG, Ayabe J, et al: Prospective clinical trial of intravitreal aflibercept treatment for polypoidal choroidal vasculopathy with hemorrhage or exudation (EPIC study): 6 month results. BMC Ophthalmol 2016;16:127.

13 Kawashima Y, Oishi A, Tsujikawa A, Yamashiro K, Miyake M, Ueda-Arakawa N, et al: Effects of aflibercept for ranibizumab-resistant neovascular age-related macular degeneration and polypoidal choroidal vasculopathy. Graefes Arch Clin Exp Ophthalmol 2015;253:1471-1477.

14 Yonekawa Y: Aflibercept for the treatment of refractory polypoidal choroidal vasculopathy. Can J Ophthalmol 2013;48:e59-e60.
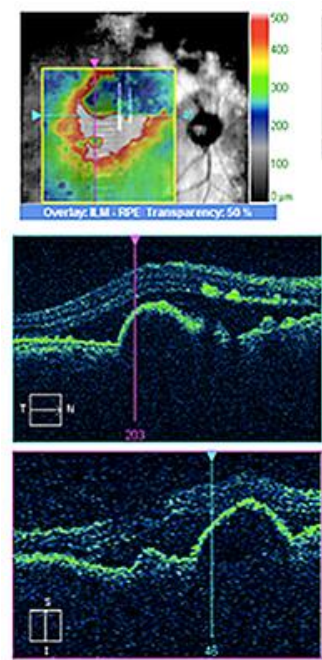

a
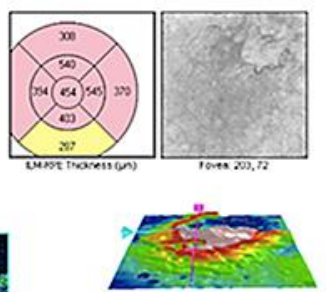

w.
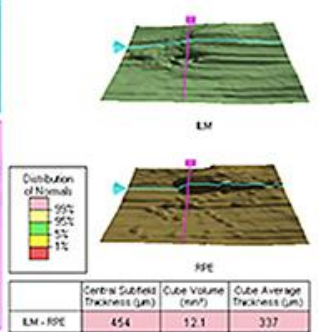
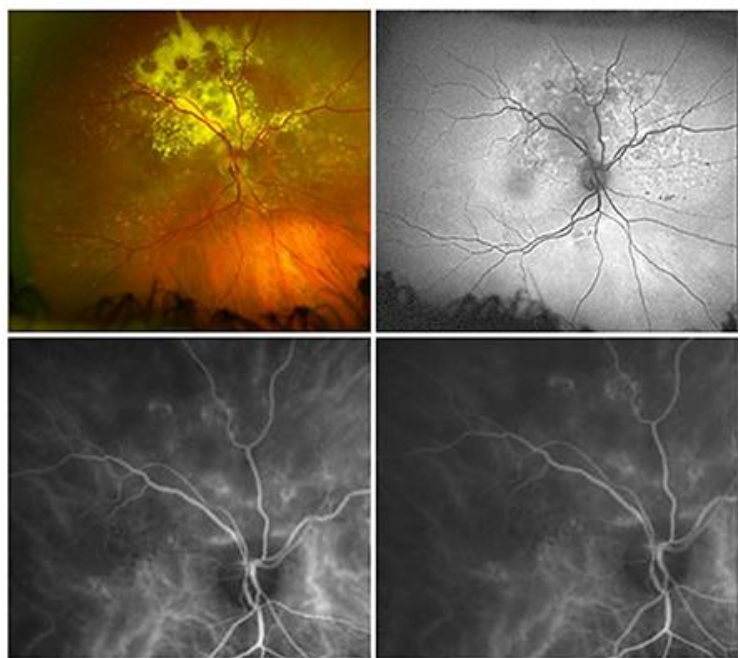

b

Fig. 1. Optical coherence tomography showing an area with subretinal fluid leakage (a) and fluorescein angiography showing the presence of significant pigment epithelial detachment in the perifoveal capillary network (venous phase) just before starting treatment with ranibizumab (b). No polyps were observed. c Indocyanine green angiography performed 3 months later (after initial loading dose of ranibizumab 0.5 $\mathrm{mg}$ ) showing 3 hyperfluorescent polyps in the superior papillary area of the right eye. 
Medina-Baena et al.: One-Year Outcome of Aflibercept and PDT in a Caucasian Patient with PCV Refractory to Ranibizumab and Photodynamic Therapy

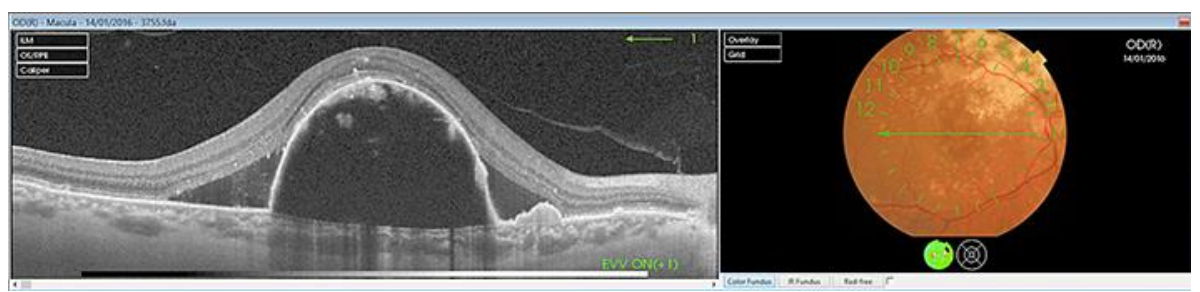

Fig. 2. Optical coherence tomography at the follow-up visit 4 weeks after combination therapy with ranibizumab $0.5 \mathrm{mg}$ and photodynamic therapy (right eye).

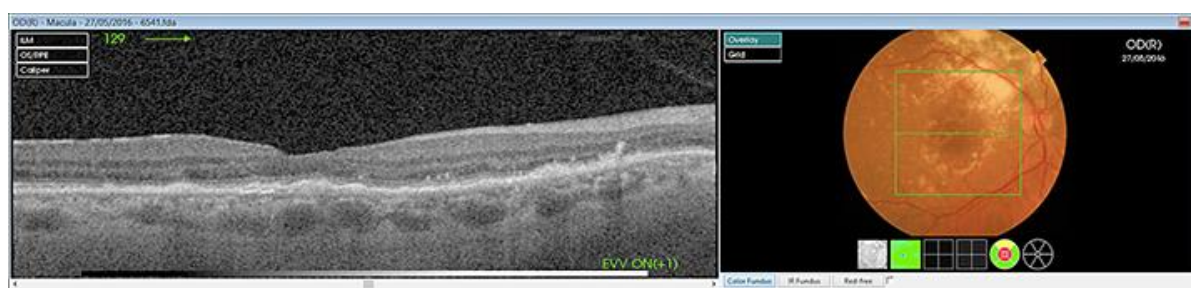

Fig. 3. Optical coherence tomography performed 4 weeks after administration of the loading dose of aflibercept followed by half-fluence photodynamic therapy (right eye). 